\title{
Planungskultur als Sinnsystem. Eine Untersuchung am Beispiel kooperativer Stadtgrünentwicklung in Düsseldorf
}

\author{
Martin Sondermann ${ }^{1}$
}

Eingegangen: 7. März 2016 / Angenommen: 31. Oktober 2016 / Online publiziert: 23. November 2016 (c) Springer-Verlag Berlin Heidelberg 2016

Zusammenfassung Wie vollzieht sich das normative Ideal kooperativer Stadtplanung in der Praxis? Am Beispiel kooperativer Stadtgrünentwicklung in Düsseldorf wird in dieser Arbeit qualitativ untersucht, wie zivilgesellschaftliche und politisch-administrative Akteure zusammenarbeiten. Dabei wird eine kulturelle Perspektive auf Planungspraxis eingenommen und die empirischen Beobachtungen mittels eines Grounded Theory-Ansatzes zu einem theoretischen Planungskultur-Modell abstrahiert. Es wird gezeigt, wie sich eine „Kultur des Miteinander” in der Düsseldorfer Stadtgrünentwicklung herausgebildet hat: Diese ist unter anderem gekennzeichnet durch eine lebendige Tradition der Zusammenarbeit und gemeinsame Orientierungen der Akteure. Sie zeigt sich beispielsweise in der Art und Weise, wie institutionelle Handlungsspielräume genutzt, organisatorische Strukturen angepasst und Verfahrensweisen verändert werden. In den theoretischen Schlussfolgerungen wird ein Verständnis von Planung aufgezeigt, welches die spezifischen Aushandlungsprozesse auf lokaler Ebene betont. Im Ergebnis werden lokale Planungskulturen als Sinnsysteme beschrieben, die sich kontextspezifisch durch Kommunikations-, Lern- und Annäherungsprozesse zwischen den beteiligten Akteursgruppen über die Zeit herausgebildet haben, deren Handeln leiten und sich in den Arten und Weisen der sozialen Raumproduktion widerspiegeln.

Schlüsselwörter Planungstheorie · Planungspraxis · Planungskultur · Kooperation · Zivilgesellschaft · Stadtgrün

Dipl.-Geogr. Martin Sondermann sondermann@arl-net.de

1 Akademie für Raumforschung und Landesplanung (ARL), Hohenzollernstr. 11, 30161 Hannover, Deutschland

\section{Planning Culture as a System of Meaning. A Study Using the Example of Cooperative Green Urban Development in Düsseldorf, Germany}

\begin{abstract}
How is the normative ideal of cooperative urban planning working in practice? Taking the case of green urban development in Düsseldorf (Germany) the analysis is focused on the cooperation between civil society and political-administrative actors. Thereby planning practice is qualitatively analysed from a cultural perspective. The empirical observations are theorised based on a Grounded Theory approach leading to a model of planning culture. It is shown how a 'culture of cooperation' has emerged in Düsseldorf's green urban development, including a living tradition of cooperation and shared orientations of the actors. This culture is observable in the institutional scopes of actions and the adaptation of organisational structures and procedures. The theoretical output of this research illustrates an understanding of planning which emphasises the specific forms of negotiating spatial development on the local level. Ultimately local planning cultures are described as context-specific systems of meaning, which have emerged over time through processes of communication, learning and rapprochement between the actors involved. These systems of meaning guide planning-related actions and manifest in the ways spaces are socially produced.
\end{abstract}

Keywords Planning theory $\cdot$ Planning practice $\cdot$ Planning culture $\cdot$ Cooperation $\cdot$ Civil society $\cdot$ Urban green spaces

\section{Zur Kultur kooperativer Stadtentwicklung}

Eine zeitgemäße Stadtentwicklung basiert auf integrierten Ansätzen, kooperativen (Governance-)Strukturen und kom- 
munikativen Aushandlungsprozessen - so zumindest die idealtypische Vorstellung in Planungswissenschaft, -politik und -praxis (vgl. Nuissl/Heinrichs 2011; Healey 2012; DST 2013). Zudem sollen insbesondere auch zivilgesellschaftliche Akteure durch die planenden Verwaltungen in konkrete Projekte mit eingebunden bzw. es sollen ihre Bottom$u p$-Initiativen unterstützt werden (vgl. BMVBS/BBR 2007: 17 f.; Selle 2007). Allerdings gestaltet sich die kooperative Zusammenarbeit von öffentlichen und zivilgesellschaftlichen Akteuren in der Praxis häufig schwierig - sei es aufgrund von Interessenkonflikten, der (Nicht-)Repräsentation bestimmter Gruppen, Fragen der Legitimation direktdemokratischer Verfahren oder der ungleichen Verteilung von Ressourcen und Macht. Hier kann von einer generellen Diskrepanz zwischen planungstheoretischen Ansprüchen und planungspraktischen Realitäten ausgegangen werden (vgl. Reuter 2000; Marx 2007; Selle 2007; Siebel 2009).

Um Theorien von räumlicher Planungspraxis zu entwickeln, welche möglichst nahe an der Realität der Akteure sind, bedarf es Ansätzen, die die spezifischen nationalen, vor allem aber auch lokalen (gesellschaftlichen, politischen) Kontexte berücksichtigen und Planungspraktiken in ihren jeweils charakteristischen Merkmalen abbilden (vgl. Othengrafen/Reimer 2013: 1280 f.). Hierzu werden in der Planungswissenschaft derzeit analytische Planungskulturansätze diskutiert, welche in ihrer empirischen Anwendung zu neuen Theorien von räumlicher Planung beitragen sollen (vgl. Othengrafen/Reimer 2013; Levin-Keitel/Sondermann 2015).

Als empirischer Untersuchungsbereich dient in diesem Beitrag die kooperative Stadtgrünentwicklung, also die Zusammenarbeit von zivilgesellschaftlichen und politischadministrativen Akteuren im Handlungsbereich urbaner Grünräume, welche aufgrund aktueller Gartenbewegungen jüngst wieder in der raumwissenschaftlichen Forschung diskutiert wird (Rosol 2010; BBSR 2015; Mackrodt 2015; Ioannou/Moran/Sondermann et al. 2016; Nikolaïdou/Klöti/ Tappert et al. 2016). Die Stadtgrünentwicklung ist besonders interessant für eine Untersuchung, da sie in der Praxis als ein kooperatives Handlungsfeld verstanden wird, aber im Vergleich zu anderen kooperativen Handlungsfeldern (insbesondere im Kontext von Stadtumbau und dem Programm ,Soziale Stadt") bisher kaum als solches untersucht wurde. Als Fallbeispiel wurde Düsseldorf ausgewählt, da die Stadt als gutes Beispiel integrierter und kooperativer Stadtgrünentwicklung identifiziert wurde (vgl. Bläser/FoxKämper/Rawak et al. 2012).

Ziel dieses Beitrags ist es, aus einer kulturellen Perspektive heraus die lokale Praxis kooperativer Stadtentwicklung an diesem Exempel zu untersuchen und dabei einen empirisch fundierten, theoretischen Beitrag zur Planungskulturforschung zu leisten. Die dabei zugrunde liegenden Überlegungen zur Planungskultur (Kapitel 2) werden mit- tels eines Grounded Theory-Ansatzes (Kapitel 3) und der Fallstudienanalyse in ein empirisch fundiertes Modell zur Analyse und Darstellung lokaler Planungskultur überführt. Anschließend werden die empirischen Merkmale und $\mathrm{Zu}$ sammenhänge der Düsseldorfer Planungskultur kooperativer Stadtgrünentwicklung anhand dieses Modells herausgearbeitet (Kapitel 4), resümiert und abstrahiert (Kapitel 5).

\section{Der „Cultural Turn“ in der Planungswissenschaft}

Räume sind nicht gegeben, sie werden gemacht. Diese Aussage gehört wohl zu den grundlegendsten Annahmen, die raumplanerischem Handeln zugrunde liegt. So kennzeichnet sich planerisches Handeln durch die Arten und Weisen, wie auf räumliche Entwicklungen Einfluss genommen wird, Belange und Interessen abgewogen, koordiniert, kommuniziert und entschieden werden. Dabei wird die kulturelle Beschaffenheit des Planens auf zwei Ebenen - gedanklich und im praktischen Vollzug - deutlich:

Auf einer grundlegenden Ebene können Räume als Ergebnisse gesellschaftlicher Praxis und damit als Ausdruck von Kultur gedacht werden. Ein derartiges, relationales bzw. sozial-konstruktivistisches Raumverständnis korrespondiert mit dem „cultural turn“ in den Sozialwissenschaften und der Humangeographie, wie er sich in der deutschsprachigen Debatte vor allem in den 1990erJahren vollzog (vgl. Läpple 1992; Löw 2001; Lossau 2007). Analog hierzu wird auch das räumliche Planen explizit als kulturelles Handeln gedeutet und dabei der Begriff Planungskultur in die planungswissenschaftliche Debatte eingeführt (Keller/Koch/Selle 1993a; Keller/Koch/ Selle 1993b; Friedmann 2005; Fürst 2007: 2; Othengrafen/ Reimer 2013: 1271). Was die meisten der derzeit diskutierten Planungskulturverständnisse eint, ist die Annahme, dass räumliche Planung in ihrer Gesamtheit Ausdruck von Kultur ist (Knieling/Othengrafen 2009; Levin-Keitel/ Sondermann 2015). Dies entspricht einem holistischen Kulturverständnis, nach dem Kultur eine „universelle Ordnung “ ist, welche im Allgemeinen das gesamte menschliche Denken und Handeln umfasst und im Speziellen in definierten Kontexten jeweils spezifisch ausgeprägt ist (vgl. Riegler 2003: 8; Reckwitz 2005: 95; Othengrafen/Reimer 2013; Peer/Sondermann 2016).

Um die spezifischen Ausprägungen zu erkennen und zu verstehen, bedarf es empirischer Beobachtungen des praktischen Vollzugs einer Kultur (vgl. Peer/Sondermann 2016). Dabei wird gefragt, wie bestimmte Deutungsweisen, Regeln oder auch Artefakte erschaffen, angewandt oder verändert werden (vgl. Hetzel 2001: 10 f.). Die spezifischen Handlungs- und Interaktionsweisen der an einer Planungskultur beteiligten Akteure korrespondieren mit bestimmten 
Denkweisen, welche das Handeln leiten und in ihrer Gesamtheit ein Sinnsystem bilden. Dieses Sinnsystem wird verstanden als ein System, mit dem ,sich die Handelnden ihre Wirklichkeit als bedeutungsvoll erschaffen und die in Form von Wissensordnungen ihr Handeln ermöglichen und einschränken“ (Reckwitz 2000: 84).

Ziel einer empirischen Erforschung von Planungskulturen ist das Verstehen und Erklären von

- nationalen, regionalen und lokalen Unterschieden in den Arten und Weisen des Planens (Othengrafen 2010; Levin-Keitel 2015),

- Rollen und Selbstverständnissen von Planerinnen und Planern (Cultplan 2007),

- Prozessen des Wandels planerischer Praktiken (Reimer 2013) und

- Abhängigkeiten räumlichen Planens von gesellschaftlichen und politischen Kontexten (Haumann 2015; Hölzl/ Nuissl 2015).

Dabei werden sowohl vergleichende Ansätze (z. B. Sanyal 2005; Knieling/Othengrafen 2009; Reimer/Blotevogel 2012) als auch vertiefende Einzelfallstudien durchgeführt (z. B. Suitner 2014; Hölzl/Nuissl 2015), je nachdem ob es programmatisch um die Herausarbeitung von Gemeinsamkeiten und Unterschieden durch Vergleiche oder um die Verortung von Planungskulturen in spezifischen gesellschaftlichen Kontexten geht (vgl. auch Peer/Sondermann 2016).

In dieser Arbeit wird Planungskultur verstanden als ein analytischer Ansatz zur Untersuchung spezifischer Formen räumlichen Planens, die sich durch die Denk- und Handlungsweisen der beteiligten Akteure in Abhängigkeit von deren Handlungskontexten vollziehen und sich in der sozialen Produktion von Räumen manifestieren. Dieser Planungskulturbegriff wird im Folgenden als theoretischer $\mathrm{Zu}-$ gang zur Erforschung einer empirisch beobachtbaren Planungskultur angewandt. Basierend auf dieser Untersuchung wird mittels eines Grounded Theory-Ansatzes ein konkretes Planungskulturmodell entwickelt.

\section{Vorgehensweise, Methodik und Modell}

Um Planungskultur(en) empirisch zu untersuchen, bedarf es geeigneter analytischer Modelle, welche ein Verständnis von Planung in entsprechende Betrachtungsebenen und Betrachtungselemente übersetzen, also operationalisieren, sowie Sinnzusammenhänge sichtbar machen. Verschiedene theoretisch hergeleitete Modelle wurden in den letzten Jahren erarbeitet, die Planungskulturen strukturell und prozedural erklären (Othengrafen 2012; Reimer 2013; Hölzl/ Nuissl 2015). Diese Ansätze haben ihren epistemologischen Ursprung in kulturwissenschaftlichen Ansätzen, beispiels- weise zur Untersuchung von Organisationskultur (vgl. Othengrafen 2012: 56 ff.; Levin-Keitel/Sondermann 2015: 49 f.). Sie wurden also aus anderen Disziplinen auf den Forschungsbereich der Planungswissenschaft übertragen und nicht originär innerhalb der Disziplin gebildet. Alternativ hierzu wird in dieser Arbeit eine Forschungsstrategie verfolgt, welche es ermöglicht, aus der Planungswissenschaft heraus selbst ein theoretisches Modell zu entwickeln, um Planungskulturen empirisch zu untersuchen und qualitativ zu interpretieren.

\section{1 „Grounded Theory“ als forschungsleitende Strategie, Methodik und Vorgehensweise}

Grundlage der forschungsleitenden Strategie ist die auf Glaser und Strauss (1967) zurückgehende Grounded Theory. Diese ermöglicht eine reflektierte Theoriebildung, welche aus der Empirie heraus erfolgt bzw. in dieser verankert (,grounded“) ist (vgl. Strauss/Corbin 2010). Die Theoriebildung erfolgt durch einen Wechsel zwischen induktivem und deduktivem Denken: Beobachtete Phänomene werden abstrahiert, also theoretische Konstrukte gebildet und diese wiederum anhand der beobachteten Phänomene (Empirie) überprüft. Diese explorative und qualitative Vorgehensweise ermöglicht die Aufstellung einer neuen Theorie mittlerer Reichweite aus einer gegenstands- und fallspezifischen Empirie heraus. Ihre Aussagekraft bezieht sich in diesem Beitrag gegenständlich auf Stadtgrünentwicklung am Fallbeispiel von Düsseldorf und ist in ihrer Abstraktion sowie durch weitere empirische Forschung auch auf andere Handlungsbereiche und Handlungskontexte räumlichen Planens übertragbar.

Im Fokus der Untersuchung stehen die Akteure der kooperativen Stadtgrünentwicklung (vgl. Abbildung 1): Hierzu gehören erstens die zivilgesellschaftlichen Akteure, in diesem Fall Vereine und nicht institutionalisierte Gruppen engagierter Bürgerinnen und Bürger. Die zweite Gruppe wird als politisch-administrative Akteure angesprochen, wozu im konkreten Fall das Stadtplanungs-, das Umwelt- und das Gartenamt der Landeshauptstadt Düsseldorf gehören. Weitere potenziell involvierte Gruppen sind intermediäre, vermittelnde Institutionen wie Quartiersmanagements sowie Grundstückseigentümerinnen und -eigentümer. Allerdings spielten diese beiden Gruppen in den untersuchten Projekten in Düsseldorf keine erkennbare Rolle, weshalb die Auswahl im Forschungsprozess auf die beiden zuerst genannten Akteursgruppen beschränkt wurde.

Entsprechend des hergeleiteten Planungskulturverständnisses (vgl. Kapitel 3.2) liegt der Fokus der empirischen Untersuchung auf den Denk- und Handlungsweisen der beteiligten Akteure. Um diese methodisch zu erschließen, wurden insgesamt acht Interviews von je etwa 50 Minuten geführt, jeweils vier mit zivilgesellschaftlichen und politisch- 
Abb. 1: Akteure der kooperativen Stadtgrünentwicklung
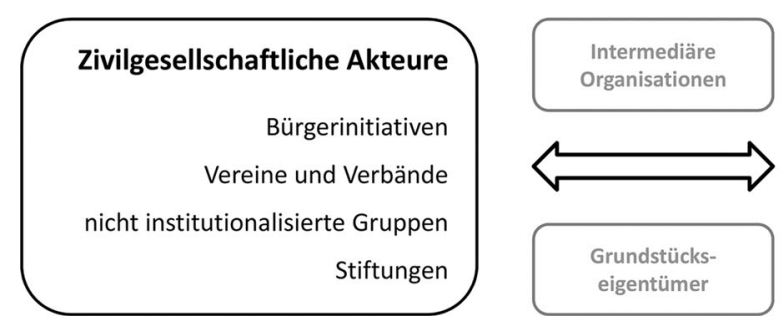

Politisch-administrative Akteure

Stadt- und Stadtbezirksräte

Verwaltungs-Fachbereiche für Umwelt, Stadtgrün, Stadtplanung und -entwicklung

Tab. 1: Verzeichnis der Akteursinterviews

\begin{tabular}{llll}
\hline Akteursgruppe & CODE* & Datum & Länge \\
\hline Stadtverwaltung & DV1 & 26.11 .2013 & $00: 59$ \\
(Gartenamt, Umweltamt, Stadtplanungsamt) & DV2 & 26.11 .2013 & $01: 06$ \\
& DV3 & 27.11 .2013 & $00: 40$ \\
Zivilgesellschaftliche Akteure & DV4 & 28.11 .2013 & $01: 01$ \\
(Vereine, nicht-institutionalisierte Gruppen) & DZ1 & 25.11 .2013 & $00: 50$ \\
& DZ2 & 26.11 .2013 & $00: 34$ \\
& DZ3 & 29.11 .2013 & $00: 42$ \\
\hline
\end{tabular}

* Code: $\mathrm{D}$ = Düsseldorf; $\mathrm{V}=$ Verwaltung; $\mathrm{Z}=$ Zivilgesellschaft

Eigene Darstellung

administrativen Akteuren (vgl. Tabelle 1). Dabei wurden alle wesentlichen Akteure von kooperativen, gärtnerischen Stadtgrünprojekten interviewt, die auf öffentlichen Grundstücken realisiert und im Zeitraum der Untersuchung aktiv betrieben wurden. Diese Auswahl ermöglicht dabei eine aussagekräftige Analyse der Fallstudie Düsseldorf. Die Interviews waren leitfadengestützt, teilstandardisiert und ermöglichten auch längere narrative Ausführungen der Interviewpartner sowie ein flexibles Nachfragen (vgl. Wessel 1996: 132 f.; Hopf 2004: 353 ff.).

Die vollständig in Schriftdeutsch transkribierten Interviews wurden entsprechend der Grounded Theory in Form einer theoretischen Kodierung ausgewertet. Die Auswertung umfasste im Wesentlichen folgende Schritte: Zunächst wurden alle Interviews offen kodiert, also die Transkripte in Sinnabschnitte gegliedert, diese bezeichnet und anschließend induktiv zu Kategorien zusammengefasst (vgl. Strauss/Corbin 2010: 43 ff.). Anschließend wurde ein System von Ober- und Unterkategorien erarbeitet, dieses in Sinnzusammenhängen gefasst (axial kodiert) und dabei auf das sogenannte Kodierparadigma zurückgegriffen (vgl. Tabelle 2; vgl. Strauss/Corbin 2010: 75 ff.).

Mit der Anwendung des Kodierparadigmas wird auch das Ziel der Anwendung der Grounded Theory deutlich, nämlich die Entwicklung eines Modells, welches Planungskultur aus den empirisch beobachteten Sinnzusammenhängen heraus erklärt. Diese Sinnzusammenhänge bilden die Handlungen der an der kooperativen Stadtgrünentwicklung beteiligten Akteure hinsichtlich der ursächlichen Bedingungen, Kontexte, Strategien, intervenierenden Bedingungen und Konsequenzen ab (vgl. Tabelle 2; vgl. Strauss/Corbin
2010: 75 ff.). Der letzte Schritt bestand in der Überprüfung und iterativen Überarbeitung des Kategoriensystems am empirischen Material. Diese Überarbeitung wurde insgesamt fünfmal wiederholt, bis sich ein konsistentes Bild ergab, also die Kategorien alle relevanten inhaltlichen Aussagen aus den Interviews abdeckten und sich in einem in sich logischen System entsprechend des Kodierparadigmas einordnen ließen (vgl. Abbildung 2 und Tabelle 3).

\subsection{Modell: Planungskultur als Sinnsystem}

Im Fokus der Betrachtung steht das empirisch beobachtete Phänomen der kooperativen Zusammenarbeit zivilgesellschaftlicher und administrativer Akteure in der Stadtgrünentwicklung (vgl. Abbildung 2 und Tabelle 3): Dieses Phänomen basiert ursächlich darauf, dass es Initiativen und Engagement zur kooperativen Zusammenarbeit gibt, sowie auf den damit verbundenen Motiven und Zielen der Akteure. Die kooperative Zusammenarbeit selbst wird durch den institutionellen Handlungsspielraum gewissermaßen begrenzt, da die Akteure nicht frei von politischen Entscheidungen und Gesetzen agieren. Als sichtbare Artefakte der Zusammenarbeit entstehen Pläne und Konzepte sowie kooperativ geplante und/oder realisierte Stadtgrünprojekte. Im Verlauf der Zusammenarbeit handeln die beteiligten Akteure die Raumnutzung kommunikativ aus, lernen sich dabei kennen und nähern sich gegebenenfalls in ihren Denkweisen an. Diese Kommunikations-, Lernund Annäherungsprozesse werden wiederum durch die handlungsleitenden Denkweisen intervenierend beeinflusst, wozu beispielsweise die Offenheit gegenüber anderen Ak- 
Tab. 2: Das Kodierparadigma der Grounded Theory

\begin{tabular}{ll}
\hline Kategorie & Erläuterung \\
\hline Phänomen & $\begin{array}{l}\text { Die zentrale, beobachtete Erscheinung, auf die sich die Handlungen und Interaktionen } \\
\text { beziehen }\end{array}$ \\
Ursächliche Bedingungen & $\begin{array}{l}\text { Bedingungen, die zum Auftreten oder der Entwicklung des Phänomens führen } \\
\text { Eigenschaften, die zu einem Phänomen gehören und den Handlungs-/Interaktionsrahmen } \\
\text { Kontext }\end{array}$ \\
bilden \\
Intervenierende Bedingungen & $\begin{array}{l}\text { Auf das Phänomen gerichtete Handlungen und Interaktionen von Akteuren } \\
\text { Bedingungen, die auf Handlungen und Interaktionen einwirken und diese fördern oder } \\
\text { hemmen }\end{array}$ \\
Konsequenzen & Ergebnisse der Handlungen und Interaktionen \\
\hline
\end{tabular}

Eigene Darstellung (nach Strauss/Corbin 2010: 75-85).

Abb. 2: Lokale Planungskultur als Sinnsystem (am Beispiel kooperativer Stadtgrünentwicklung) Quelle: Eigene Darstellung in Anlehnung an Strauss/ Corbin (2010: 75 ff.)

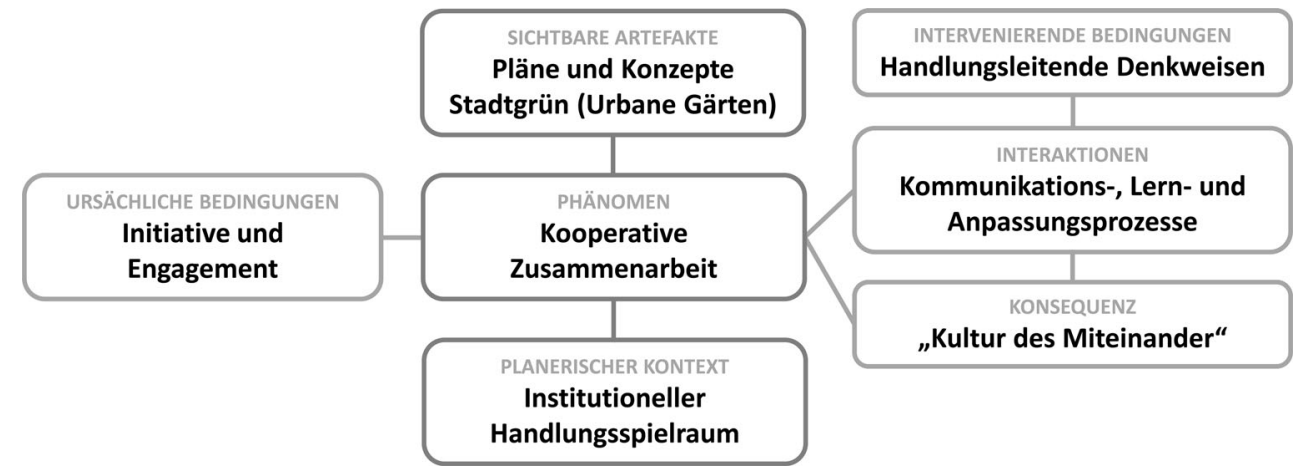

teuren gehört. In der Konsequenz entsteht im betrachteten Fall eine „Kultur des Miteinander”, welche sich unter anderem durch ein vertrauensvolles Verhältnis der Akteure mit wechselseitigem Verständnis und gegenseitiger Anerkennung kennzeichnet. Dies wiederum beeinflusst die Art und Weise der kooperativen Zusammenarbeit bzw. kennzeichnet diese.

\section{Planungskultur als Sinnsystem - das Fallbeispiel kooperative Stadtgrünentwicklung in Düsseldorf}

Im Folgenden wird das aus der Empirie heraus entwickelte Planungskulturmodell (Abbildung 2 und Tabelle 3) wiederum auf die empirischen Ergebnisse der Untersuchung angewandt, also das Fallbeispiel der kooperativen Stadtgrünentwicklung in Düsseldorf entsprechend der Kategorien und Elemente des Modells in seinen konkreten Ausprägungen dargestellt.

\subsection{Sichtbare Artefakte}

In Düsseldorf gibt es eine Vielzahl von Kooperationsformen zwischen zivilgesellschaftlichen und politisch-administrativen Akteuren in der Stadtgrünentwicklung. Bis heute wirksam sind Ansätze kooperativer Stadtgrünentwick- lung, wie sie in Düsseldorf seit Anfang der 1980er-Jahre gelebt werden. Erwähnenswert sind hier Maßnahmen zur Wohnumfeldverbesserung, welche unter anderem die Begrünung von Baumscheiben, Innenhöfen und Dächern umfassen. Bis heute unterstützen Stellen der Stadtverwaltung (Stadtplanungs-, Umwelt- und Gartenamt) engagierte Bürgerinnen und Bürger mit ihren Begrünungsvorhaben administrativ und finanziell (DV1; DV2; DV3; DV4; LHD 70/6/2010).

Zudem gibt es in Düsseldorf mehrere zivilgesellschaftliche Garten- und Parkprojekte, von denen insbesondere das Ökotop Heerdt hervorzuheben ist: Das rund 16 ha große Areal wird seit 1986 auf einer Industriebrache kooperativ mit Stadtpolitik und Stadtverwaltung geplant und realisiert und ist bis heute in Verantwortung eines Vereins. Zugleich ist es ein frei zugänglicher Park, der Gemeinschaftsgärten, eine Ökosiedlung sowie verschiedene ökologische Lehreinrichtungen (wie einen Naturlehrpfad und eine Imkerei) beherbergt (Steffler/Funke/Potratz et al. 2006). Ein ähnliches, aber kleineres Projekt ist der Nachbarschaftsgarten „Neue Lohe“, der 1995 von einer Anwohnerinitiative auf einem ehemaligen Parkplatz angelegt wurde. Beide Projekte wurden und werden von Politik und Verwaltung unterstützt und dort bis heute wertgeschätzt (DV2; DV4; DZ1; DZ3; LHD 19/8/2012; LHD 70/8/2012). Aktuell gibt es einen Gemeinschaftsgarten in der Haifastraße von der Initiative ,düsselgrün“, der von Politik und Verwaltung als Zwischennutzung 
Tab. 3: Kategorien und Elemente des Planungskultur-Modells

\begin{tabular}{|c|c|c|}
\hline $\begin{array}{l}\text { Kategorien des Kodierpara- } \\
\text { digmas* }\end{array}$ & $\begin{array}{l}\text { Kategorien des Planungskultur- Mo- } \\
\text { dells** }\end{array}$ & Betrachtungselemente \\
\hline Phänomen & Kooperative Zusammenarbeit & $\begin{array}{l}\text { - Beteiligte Akteure } \\
\text { - Akteurskonstellationen } \\
\text { - Rollenverteilungen }\end{array}$ \\
\hline Ursächliche Bedingungen & Initiativen und Engagement & $\begin{array}{l}\text { - Initiativen zur Zusammenarbeit } \\
\text { - Ausprägungen des Engagements } \\
\text { - Motivationen und Ziele }\end{array}$ \\
\hline Kontext & Institutioneller Handlungsspielraum & $\begin{array}{l}\text { - Politisch definierter Handlungsrahmen } \\
\text { - Gesetze und rechtliche Regularien } \\
\text { - Personelle und finanzielle Ressourcen }\end{array}$ \\
\hline $\begin{array}{l}\text { Handlungs- und interaktio- } \\
\text { nale Strategien }\end{array}$ & $\begin{array}{l}\text { Kommunikations-, Lern- und Annä- } \\
\text { herungsprozesse }\end{array}$ & $\begin{array}{l}\text { - Formen der kommunikativen Zusammenarbeit } \\
\text { - Lern- und Annäherungsprozesse } \\
\text { - Befördernde und hemmende Faktoren }\end{array}$ \\
\hline $\begin{array}{l}\text { Intervenierende Bedingun- } \\
\text { gen }\end{array}$ & Handlungsleitenden Denkweisen & $\begin{array}{l}\text { - Orientierungen, Einstellungen und Haltungen } \\
\text { - Traditionen }\end{array}$ \\
\hline \multirow[t]{2}{*}{ Konsequenzen } & Kultur des Miteinander*** & $\begin{array}{l}\text { - Konstruktive, offene Haltung } \\
\text { - Gemeinsame Orientierungen } \\
\text { - Gegenseitige(s) Verständnis und Anerkennung } \\
\text { - Anpassungsbereitschaft }\end{array}$ \\
\hline & Sichtbare Artefakte & $\begin{array}{l}\text { - Raumbezogene Pläne, Konzepte und Programme } \\
\text { - Planungsrelevante Dokumente } \\
\text { - (z.B. Ratsbeschlüsse) } \\
\text { Physische Artefakte (z.B. Gemeinschaftsgärten) }\end{array}$ \\
\hline
\end{tabular}

* Kategorien des Kodierparadigmas nach Strauss/Corbin 2010: 75-85

** Eigene Kategorien als Ergebnis axialen Kodierens des empirischen Materials

*** Fallspezifische Kategorie-Bezeichnung

Eigene Darstellung

nachträglich temporär genehmigt wurde. Dieser Garten fungierte neben dem Garten am Ausstellungshaus „Kunst im Tunnel“ (KIT) (vgl. Abbildung 3) als ein ,Satellitengarten“ des Projekts „Elisabeths Garten“ während des Kunstfestivals Quadriennale Düsseldorf 2014 (DZ1; DZ4; vgl. Stiftung Schloss und Park Benrath 2014).

Ein weiterer Gemeinschaftsgarten (Ellerstraße) wurde top-down vom Stadtplanungsamt auf einer nicht bebaubaren Baulücke initiiert und gemeinsam mit engagierten Bürgerinnen und Bürgern realisiert, die ihn heute als Verein „Oberbilker Stadtgärtner“ ehrenamtlich betreiben (Landes-

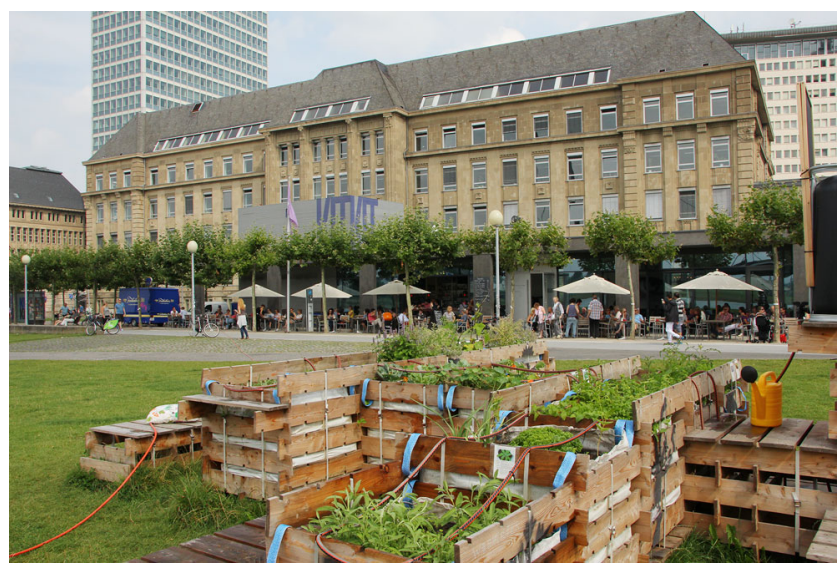

Abb. 3: Satellitengarten am KIT 2014 (Projekt Elisabeths Garten) hauptstadt Düsseldorf 2009: 26). Letztlich ist das stadtweite Baumpatenschaftsprogramm zu nennen: In Düsseldorf dürfen Bürgerinnen und Bürger seit Anfang der 1980erJahre ohne bürokratische Verfahren Baumscheiben bepflanzen. Die Zahl der sogenannten Baumpaten wird derzeit auf 5.000 geschätzt (LHD 70/6/2010; DV1; DV4).

\subsection{Initiativen und Engagement}

Ursächliche Bedingung für (erfolgreiche) Kooperationen in der Stadtgrünentwicklung sind entsprechende Initiativen zur Zusammenarbeit, welche sowohl von zivilgesellschaftlichen Akteuren als auch von der Stadtverwaltung ausgehen können: So sind auf zivilgesellschaftlicher Seite vor allem Baumschutzgruppen, Kleingartenvereine, Selbsternte- und Gemeinschaftsgartenprojekte sowie (stadtquartierbezogene) Bürgerinitiativen für Grünflächen und Stadtbäume zu nennen (DV1; DV3; DZ1). Aus Sicht der Verwaltung ist eine ,erfolgreiche Initiierung [...] jedoch von einem starken bürgerschaftlichen Engagement und der längerfristigen Übernahme von Verantwortung durch Bürgerinnen und Bürger abhängig“ (LHD 70/8/2012: 5). Diese Einschätzung deckt sich mit denen der erfolgreichen Initiativen: „Was für uns zum Schluss eigentlich sehr befriedigend war, ist - dass wenn man genug kämpft - als Bürger eine gewisse Macht 
hat. Und wir haben uns da engagiert und wirklich versucht, das Projekt umzusetzen“ (DZ1).

Die Motivation der zivilgesellschaftlichen Akteure hat teilweise einen reaktiv-konservativen Charakter, das heißt, dass sie sich „darauf richten zu bewahren, was vorhanden ist" (DV3) oder sich mit Problemen an die Verwaltung wenden (DV4). Die ,,allermeisten“ Initiativen sind aber „konstruktiv“(DV3) und werden von den zuständigen Verwaltungen entsprechend positiv wahrgenommen und unterstützt. Auch aus der Sicht der Bürgerinnen und Bürger wird eine konstruktive Haltung als Erfolgsbedingung gesehen: „Also nur dagegen zu sein, nur zu protestieren - das bringt es nicht" (DZ1). Interessanterweise gibt es in Düsseldorf bereits seit den 1980er-Jahren ein sehr starkes zivilgesellschaftliches Engagement für das Stadtgrün, welches aber in den 1990er- und 2000er-Jahren abnahm und erst in den letzten Jahren wieder zunimmt (DV1; DV3; DZ1; DZ3). Allerdings hat sich bisher keine entsprechende Aktivisten-Szene gebildet, was darauf zurückgeführt wird, dass „Düsseldorf auch von der Bevölkerungsstruktur per se keine Stadt [ist], die solche subversiven Strukturen beflügelt" (DZ4).

Von Seiten der Stadtverwaltung gibt es seit Anfang der 1980er-Jahre verschiedene Angebote zur kooperativen $\mathrm{Zu}$ sammenarbeit, insbesondere im Bereich der Wohnumfeldverbesserung, der Lokalen Agenda 21 sowie in kommunikativen Formen der Stadt- und Grünplanung (vgl. Kapitel 4.1). Die Motivation auf der Verwaltungsseite für die Initiierung kooperativer Grünprojekte ist dabei Ausdruck eines umfassenderen Umweltbewusstseins in Politik und Verwaltung sowie einer partizipativen Grundhaltung, wie sie sich in Düsseldorf in den 1980er-Jahren entwickelte. Diese Haltung ist Teil eines sich damals bereits wandelnden Planungsverständnisses hin zu integrierten und kooperativen Ansätzen (DZ1; DZ2; DZ4; vgl. auch Kapitel 1 sowie Lange 1987).

\subsection{Institutioneller Handlungsspielraum}

Die Verwaltungsmitarbeiter ,unterstützten Bürgerengagement natürlich nicht bedingungslos, sondern in bestimmten Grenzen“ (DZ2). Jedes Handeln findet in einem gewissen Rahmen statt, der durch verschiedene Faktoren erweitert oder limitiert wird. Grundsätzlich ist das Verwaltungshandeln vom politisch definierten Handlungsrahmen geprägt. Sei es über die strategische Ausrichtung oder über die rechtsverbindliche Raumnutzung - „Entscheiden wird es immer die Politik" (DZ1). Sowohl auf kommunaler (Stadtrat) als auch auf lokaler Ebene (Bezirksrat) werden zivilgesellschaftliche Projekte bzw. die Einbeziehung von Bürgerinnen und Bürgern in Düsseldorf unterstützt (DV2; DZ1; DZ2; DZ3). Dies zeigt sich auch in den Anfragen und Anträgen aller politischen Fraktionen an die Verwaltung, die sich mit den positiven Effekten zivilgesellschaftlicher
Garteninitiativen beschäftigen (SPD; LHD 19/57/2012), diese allgemein fördern wollen (Grüne, LHD 70/39/2012) oder konkret die Bereitstellung von Grundstücken für „Urban Gardening“ unterstützten (CDU/FDP/Grüne; LHD 176/221/2015).

Dies ist insofern von zentraler Bedeutung, als dass die Verwaltung einen entsprechend weiten Handlungsspielraum hat, um mit zivilgesellschaftlichen Akteuren in der Stadtgrünentwicklung zusammenzuarbeiten. Diese ,strategischpolitische“ (DV3) Ausrichtung von Politik und Verwaltung auf eine kooperative Stadtgrünentwicklung zeigt sich in den konkreten, kooperativ umgesetzten Projekten als sichtbare Artefakte der Planungskultur (vgl. Kapitel 4.1). Politische Entscheidungen können aber auch limitierend auf den Handlungsspielraum der Verwaltung wirken. Hier sind vor allem die Prioritätensetzung bei der Realisierung bestimmter räumlicher Maßnahmen sowie Sparmaßnahmen und Renditeerwartungen bei der Grundstücksnutzung zu nennen, welche sich allerdings nicht konkret auf die beobachteten Projekte auswirken (DV2; DV3; DV4).

Der zweite bestimmende Faktor des institutionellen Handlungsspielraums sind personelle, zeitliche und finanzielle Ressourcen der beteiligten Akteure. So muss die Verwaltung heute ,,viel stärker auf die Kosten achten [...] und auf die Umsetzung ihrer Pflichtprojekte“, weshalb ,das Korsett [...] für die Verwaltung auch viel enger geworden“" ist (DV2). Die finanzielle Ausstattung der Ämter in Düsseldorf ist zwar von Sparvorgaben geprägt, aber ausreichend, um zivilgesellschaftliche Projekte zu unterstützen, teilweise unter Verwendung von Mitteln des Bund- und LänderProgramms „Soziale Stadt“ (DV3; DV4; Landeshauptstadt Düsseldorf 2009). Auch für die zivilgesellschaftlichen Gruppen spielen Ressourcen eine wichtige Rolle: Sowohl in der Planung, Umsetzung und Pflege des Grüns als auch in der Zusammenarbeit mit der Verwaltung müssen viele personelle Ressourcen aufgebracht werden (DZ1; DZ2; DZ3). „Es gab ja wirklich dieses ehrenamtliche Engagement, was das überhaupt erst möglich gemacht hat. Es war auch wirklich viel Arbeit. Es war auch viel Bürokratie, die auch erstmal überwunden werden musste“ (DZ3).

\subsection{Kommunikations-, Lern- und Annäherungsprozesse}

Die angesprochene Bürokratie verweist auf die oftmals langwierigen Aushandlungsprozesse zwischen beiden Parteien sowie auf organisatorische Strukturen, Zuständigkeiten und Verfahrensweisen des politisch-administrativen Systems. So erscheint es teilweise „undurchsichtig“ (DZ4), wer in der Verwaltung für was zuständig ist, welche Stelle eine Nutzung genehmigen kann und welche Rolle die Rats- und Bezirkspolitik dabei spielt (DZ1; DZ2; vgl. auch BBSR 2015: 55). Zudem gilt: „Man muss immer Glück 
haben, dass man einen, der auch etwas entscheiden kann, kriegt. Und dann muss man noch viel Muße mitbringen, weil die Mühlen der Stadt langsam mahlen“ (DZ4).

So zeigen sich die zivilgesellschaftlich Engagierten teilweise frustriert von langwierigen Aushandlungs- und Entscheidungsprozessen: „Das dauert ja auch, bis da was rauskommt" (DZ3; vgl. auch DV4; DZ4). Die Dauer von Verfahren wird dabei insbesondere von drei Faktoren bestimmt. Erstens bedingen die organisatorischen Strukturen der Verwaltung, dass zur Umsetzung neuer Ideen zunächst die Zuständigkeiten geklärt und verwaltungsintern abgestimmt werden müssen. Zweitens bedarf es der politischen Genehmigung durch den Stadt- oder Bezirksrat; die Verfahrensdauer ist also abhängig von der Dauer einer politischen Beschlussfassung. Drittens gilt es Veränderungen in der räumlichen Nutzung durch Instrumente planungsrechtlich zu sichern und einen Nutzungsvertrag zu schließen (LHD 70/5/2015; DV2; DV4; DZ3; DZ4).

Kennzeichnend für eine Planungskultur sind vor allem Veränderungen aufgrund von Lern- und Annäherungsprozessen. So ist zu beobachten, dass die Verwaltungsstrukturen und Verfahrensweisen in den letzten Jahren angepasst wurden - zugunsten einer effizienteren Zusammenarbeit mit den zivilgesellschaftlichen Akteuren. So wurde beispielsweise vom Ausschuss für öffentliche Einrichtungen im Februar 2015 einstimmig beschlossen, die ,Nutzung oder Zwischennutzung von Flächen durch ,urbanes Gärtnern “ in Form von Gemeinschaftsgärten“ (LHD 70/1/2015: 1) zu unterstützen, indem das Gartenamt die verwaltungsinterne Koordination übernimmt, einen zentralen Ansprechpartner benennt sowie einen Muster-Nutzungsvertrag öffentlich bekannt gibt und die Flächenbereitstellung reguliert (vgl. LHD 70/1/2015; LHD 70/5/2015).

Auch auf zivilgesellschaftlicher Seite sind Lern- und Annäherungsprozesse zu beobachten. Basierend auf ihren Erfahrungen weisen etablierte Gruppen klare Organisationsstrukturen, eine Rechtsform (in der Regel als eingetragener Verein), institutionalisiertes Engagement und ein hohes $\mathrm{Maß}$ an fachlichem und organisatorischem Wissen auf. Das Verhältnis zur Verwaltung hat sich zudem im Laufe der Zeit gefestigt und ist geprägt von einer „Kultur des Miteinander” (vgl. DV1; DV4; DZ1).

Was Politik und Verwaltung angeht, haben auch Proteste gegen Baumfällungen (z. B. Himmelgeister Kastanie, Bäume in der Altstadt) in der Verwaltung dazu geführt, dass die Routinen der Bürgerbeteiligung grundsätzlich hinterfragt und neue Sicht- und Verfahrensweisen entwickelt wurden (DV3; DV4). Dabei sind die Bürgerproteste in Politik und Verwaltung ,weit in das Bewusstsein gedrungen“ (DV1). Mit anderen Worten: ,Und es ist durchaus so wie eigentlich bei den allermeisten Demonstrationen: in einer Demokratie demonstriert man oft für das nächste Mal. Selbst wenn man dieses Mal etwas nicht verhindern konnte, kann man davon ausgehen, dass der Anker im Kopf gesetzt ist. [...] Ich glaube insgesamt die Frage, Wie binde ich denn die Interessengruppen mit ein?`, die wird immer [!] mitgedacht - und das war früher mehr zufällig: Dem einen lag das und dem anderen nicht und so weiter. Aber heute bringt man keinen größeren Prozess mehr auf die Beine, wo man das nicht mitdenkt. Und das ist schon eine echte Veränderung in den letzten zwei Jahrzehnten“ (DV3).

Neben den zivilgesellschaftlichen Initiativen haben also auch Proteste - über den jeweils konkreten Konfliktfall hinaus - eine langfristige Wirkung auf die Planungskultur der kooperativen Stadtgrünentwicklung in Düsseldorf. Inwiefern kommunikative Aushandlungsprozesse bei konkreten Projekten positiv und konstruktiv verlaufen, hängt allerdings davon ab, wie sachlich und kompromissbereit sich beide Seiten verhalten und inwiefern sie eine gemeinsame Sprache finden bzw. die „Kodizes“ der Anderen verstehen (vgl. DV1; DV3; DV4; DZ1; DZ3).

Zentrale Faktoren der Zusammenarbeit werden also durch individuelle und insbesondere kollektive Lern- und Annäherungsprozesse zwischen und innerhalb der beteiligten Akteure bestimmt. So sind frühe Stadien der Kooperation dadurch gekennzeichnet, dass sich die Parteien zunächst einmal fremd sind. Im Laufe der Zeit lernen sich diese kennen, intensivieren und verstetigen die Zusammenarbeit, zumindest dann, wenn beide Seiten positive Erfahrungen machen oder aus Konflikten lernen. Ein fortgeschrittenes Verhältnis zwischen politisch-administrativen und zivilgesellschaftlichen Akteuren zeichnet sich dann durch einen regelmäßigen, auch informellen, Austausch sowie eine „Kultur des Miteinander” aus.

\subsection{Handlungsleitende Denkweisen und die ,Kultur des Miteinander"}

Die Kommunikations-, Lern- und Annäherungsprozesse werden durch die Denkweisen der beteiligten Akteure geleitet bzw. beeinflusst und können im Laufe der Zeit in einer gemeinsamen Geisteshaltung bezüglich der kooperativen Zusammenarbeit münden, welche sich in Düsseldorf als eine „Kultur des Miteinander” zeigt: „Am Anfang waren die Gräben tief. [...] Und inzwischen ist das echt ein sehr konstruktives, agiles Miteinander" (DV3).

Die empirische Beobachtung zeigt, dass es bestimmte Haltungen, Orientierungen und Verständnisse gibt, die einerseits das Handeln der jeweiligen Akteure bzw. Akteursgruppen leiten und anderseits maßgeblich die Art und Weise der kooperativen Zusammenarbeit prägen. Die aktuelle Haltung der Düsseldorfer Verwaltung ist dabei Teil einer gelebten Tradition (vgl. Kapitel 4.1; DV1; DV4; DZ4), welche sich in den 1980er-Jahren entwickelte und von einem Mitarbeiter des Gartenamts damals so zusammengefasst wurde: „Die bewußte Zusammenarbeit des Garten-, Friedhofs- 
und Forstamtes mit dem Bürger und die Einführung unbürokratischer Abstimmungen und Verfahrensweisen sind in den verschiedenen Bereichen der privaten Wohnumfeldverbesserung erfolgreich. [...] Der Ruf nach Grün und Unterstützung sollte immer vom Bürger ausgehen - Aufgabe der Verwaltung ist es, schnell und kooperativ zu reagieren“" (Lange 1987: 243).

Diese Haltung wurde innerhalb der drei verantwortlichen Ämter tradiert, weshalb (bis) heute die Auffassung vorherrscht, dass sie Stadtgrün mit Bürgerinnen und Bürgern gemeinsam entwickeln wollen (DV4), sei es im Rahmen der Fachplanung für Spielplätze (DV4) oder durch die Aktivierung von Bürgerinnen und Bürgern für neue Park- und Gemeinschaftsgartenprojekte im Rahmen des Programms „Soziale Stadt“ (DV2; vgl. auch Landeshauptstadt Düsseldorf 2006; Anna/Erben/Görtz 2010: 12). Für die zivilgesellschaftlichen Akteure ist diese traditionell kooperative Haltung der Verwaltung auch aktuell noch wahrnehmbar: „Das [Garten-]Amt ist auch grundsätzlich dafür, also die Verwaltungsebene unterstützt das auf jeden Fall. Auch alle anderen grünen Projekte werden da unterstützt, soweit das da möglich ist" (DZ2; vgl. auch DZ1; DZ3).

Diese Tradition stützt sich zugleich auf die Geschichte und das Selbstverständnis Düsseldorfs als „Gartenstadt“ (DV4; DZ4; Markowitz 1987; Landeshauptstadt Düsseldorf 2006), wie es auch seitens zivilgesellschaftlicher Akteure bis heute gesehen wird: „Düsseldorf sieht sich schon sehr grün. Das hat auch eine ganz große, also lange Tradition mit Parkanlagen oder überhaupt Gartenanlagen. Da gilt Düsseldorf schon so als ,Gartenstadt"“ (DZ4). Dass diese Tradition bis heute lebendig ist, liegt unter anderem daran, dass Düsseldorf bis heute ein eigenständiges Gartenamt mit eigener Grünordnungsplanung hat und Stadtgrün sowohl in der Zivilgesellschaft als auch in der Politik einen hohen Stellenwert besitzt (DV1; DV2; DV3; DV4; DZ2; DZ3). Das heißt, dass die Idee von einer „Gartenstadt“ in den organisatorischen Strukturen, über die zur Verfügung stehenden Ressourcen bis hin zu tradierten Haltungen und Orientierungen institutionalisiert wurde. Für ihre integrierte Stadtgrünentwicklung bekommt die Düsseldorfer Verwaltung auch die Anerkennung von Bürgerinnen und Bürgern sowie anderen Kommunen (DV1; DV4) und ,daraus speist sich natürlich im Rückbezug das Selbstwertgefühl und das Selbstbewusstsein des gesamten Amtes“ (DV1).

Grundsätzlich handlungsleitend für die Verwaltung sind zudem die Gemeinwohlorientierung sowie die damit verbundene Aufgabe, unterschiedliche Interessen und Belange zu koordinieren (DV2; DV3; DV4). Folglich treten vor allem dann Konflikte auf, wenn es einzelnen Personen oder Gruppen nur um die eigenen Interessen geht. So versuchen einige Anwohnerinnen und Anwohner bestimmte (insbesondere lärmintensive) Nutzungen öffentlicher Grünanlagen, z. B. durch Jugendliche, einzuschränken. Aus Sicht der
Verwaltung gilt es dabei immer, das Gemeinwohl im Blick zu behalten und aufzuzeigen, ,dass es auch andere Bedürfnisse gibt“ (DV4; vgl. DV2). Projekte, die sich speziell auf das öffentliche Interesse ausrichten, genießen hingegen eine sehr hohe Anerkennung und werden entsprechend unterstützt (DV3; DZ4). Der öffentliche Nutzen richtet sich dabei vor allem nach der öffentlichen Zugänglichkeit und der allgemeinen Nutzbarkeit von Grünanlagen, was von beiden Seiten so gesehen wird (DZ1; DV2).

Eine große Schnittmenge zwischen den Initiativen und der Verwaltung besteht hinsichtlich der Orientierungen. So sehen beide Seiten die sozialen und ökologischen Funktionen von Stadtgrün im Allgemeinen und urbanen Gärten im Besonderen (vgl. Sondermann 2014: 24 f.). Auf zivilgesellschaftlicher Seite verfolgen die Akteure ,im Prinzip auch sich so wiederholende Motive, dass wir halt die Ökologie verbessern wollen, wir wollen, dass die Menschen im Viertel wieder mehr miteinander reden, einen Treffpunkt schaffen“ (DZ2).

Dies korrespondiert mit der Sichtweise der Verwaltung auf diese Projekte: „Die urbane Agrikultur in Gestalt von Gemeinschafts- oder Nachbarschaftsgärten kann eine ökologische Bereicherung dicht bebauter Stadtquartiere bedeuten, wenn beispielsweise Baulücken oder Blockinnenbereiche genutzt und die Gärten nach ökologischen Prinzipien bewirtschaftet werden. Gärten schaffen Identität und binden die Nutzerinnen und Nutzer an ihr Stadtviertel. Sie fördern in der Regel die Kommunikation und Integration verschiedener Bevölkerungsgruppen über das gemeinsame Interesse an der Gartenarbeit" (LHD 19/8/2012: 5; vgl. auch LHD $70 / 5 / 2015$ ).

Neben gemeinsamen Orientierungen sind auch bestimmte Haltungen von zentraler Bedeutung für eine erfolgreiche Zusammenarbeit. Auf der Verwaltungsseite werden ,ernsthafte“ (DV2) und „konstruktive“ (DV3) Initiativen von engagierten Bürgerinnen und Bürgern ,respektiert“ (DV2) und ,sehr ernst genommen“ (DV2); ihre Anliegen werden „konstruktiv begleitet“ (DV3) und unterstützt (vgl. Kapitel 4.2). Umgekehrt betrachtet geht es den Bürgerinnen und Bürgern darum, dass sich die Verwaltung ,ernsthaft ihrer Anliegen annimmt und um eine Lösung bemüht ist und sie nicht als störenden Faktor im reibungslosen Ablauf der Verwaltung begreift" (DV1). Dies umfasst sowohl die Beteiligung an Stadtgrünprojekten der Verwaltung, welche als „sehr vorbildhaft“ (DZ2) empfunden wird, als auch die Reaktion auf zivilgesellschaftliche Initiativen: „Die haben uns sehr unterstützt und [...] haben das gefördert [...] und wir haben ausgezeichnete Kontakte zu denen" (DZ1). Wichtig ist dabei eine gegenseitige Wertschätzung oder auch „Achtung“ (DZ4) der Arbeit der anderen Seite.

Eine Annäherung der beiden Akteursgruppen erfolgt dabei durch kommunikative Aushandlungsprozesse (vgl. Kapitel 4.4), welche sich beispielsweise auf (planungs-) 
rechtliche Fragen zur Grundstücksnutzung oder den Ablauf von Planungs- und Genehmigungsverfahren beziehen (DV1; DV3). In Düsseldorf ist zudem eine verstetigte, vertrauensvolle Kommunikationsstruktur $\mathrm{zu}$ beobachten: „Wir sind in einem konstruktiven, beständigen Austausch, weil da immer wieder Ideen und Wünsche entstehen. Man kennt sich und versteht sich" (DV3). Wenn die Akteure eine konstruktive Haltung einnehmen, sich kennen- und schätzen lernen und sich in ihren Denkweisen annähern bzw. verstehen, dann kann von einer „Kultur des Miteinander" gesprochen werden, welche im Laufe der kooperativen Zusammenarbeit entsteht und diese zugleich charakterisiert.

\section{Resümee, Abstraktion und Fazit}

Die zentralen Erkenntnisse der Untersuchung werden nun abschließend reflektiert: Welche Merkmale und Zusammenhänge können mittels des hier aufgestellten Planungskulturmodells für die Düsseldorfer Stadtgrünentwicklung beobachtet und vom Einzelfall abstrahiert werden?

\subsection{Institutionalisierung von Denkweisen, Organisationsformen und Verfahrensweisen}

Die kooperative Zusammenarbeit funktioniert in Düsseldorf auf der Grundlage gemeinsamer Haltungen und Orientierungen der beteiligten Akteure, langjähriger Erfahrungen in der Zusammenarbeit und gelebter Traditionen (vgl. Kapitel 4.4 und 4.5). Hervorzuheben ist dabei die (traditionelle) Offenheit der Verwaltungsakteure gegenüber zivilgesellschaftlichem Engagement und gegenüber neuen Ideen und Bewegungen. So kann auf den Erfahrungen in der kooperativen Stadtgrünentwicklung mit etablierten Projekten, wie dem Ökotop Heerdt oder den Baumscheibenbegrünungen, aufgebaut und Strukturen und Verfahren können an die Bedürfnisse neuer Initiativen angepasst werden. Dabei weist die kooperative Stadtgrünentwicklung in Düsseldorf ein hohes $\mathrm{Maß}$ an Institutionalisierung auf und ist mit der Hannoveraner Planungskultur vergleichbar, die ebenfalls durch eine lebendige (Gartenstadt-)Tradition und kooperative Zusammenarbeit gekennzeichnet ist (Sondermann 2015: 108 ff.). Mit anderen Worten und bezogen auf das Verwaltungshandeln im Fallbeispiel heißt das: „Jede Verwaltung hat ihre spezielle Ausrichtung im Umgang mit zivilgesellschaftlichem Engagement - meistens historisch begründet. Das ist in Düsseldorf nicht anders" (DV1).

Abstrahiert wird hier die Bedeutung der historischen Entwicklung von Planungskulturen und insbesondere von Traditionen angesprochen (vgl. auch Reimer/Blotevogel 2012: 18; Haumann 2015). Zudem zeigt sich hier, dass sich planerische Denkweisen sowie Organisationsformen und Verfahrensweisen über die Zeit institutionalisieren, also verfestigen und selbstwirksam (oft unhinterfragt) das Handeln leiten (vgl. auch Reimer 2013).

\subsection{Spezifische Interpretationen von Handlungskontexten und -möglichkeiten}

Bezüglich des institutionellen Handlungsspielraums ist festzustellen, dass die kooperative Stadtgrünentwicklung in Düsseldorf sowohl von der Politik als auch von der Verwaltung rechtlich, organisatorisch und teilweise auch finanziell unterstützt wird. Die politischen Debatten und Beschlüsse sowie organisatorische Neuregelungen belegen, wie flexibel politisch-administrative Akteure agieren (vgl. Kapitel 4.3). Daraus lässt sich folgern, dass (politische, rechtliche) Handlungskontexte sowie Ressourcen nicht deterministisch wirken, sondern als Handlungsspielräume $\mathrm{zu}$ interpretieren sind. In diesem Sinne fungieren sie in der Planungskulturforschung als ein Spiegel, in dem sich die handlungsleitenden Denkweisen betrachten lassen und beispielsweise gefragt werden kann: Wie gehen Verwaltungsakteure mit knappen Budgets um? Wo werden Prioritäten gesetzt? Mit welchen Mitteln werden Projekte finanziert? Und was sagt dies über die vorherrschenden Denkweisen, die Wertschätzung zivilgesellschaftlichen Engagements und das eigene Rollenverständnis aus? Kurzum betont dieses Planungskulturmodell, dass Handlungskontexte und -möglichkeiten von den beteiligten Akteuren immer spezifisch interpretiert werden (vgl. auch Knieling/ Othengrafen 2015: 2135).

\subsection{Lern- und Annäherungsprozesse}

Die dabei handlungsleitenden Denkweisen sind im Fallbeispiel dadurch charakterisiert, dass beide Seiten eine konstruktive Haltung und gemeinsame Orientierungen einnehmen und für das Handeln der anderen Seite Verständnis und Anerkennung aufbringen, wodurch eine „Kultur des Miteinander" entstanden ist (vgl. Kapitel 4.5 sowie Sondermann 2014: 28 f.). Hierbei spielen Erfahrungen und gemeinsame Lern- und Annäherungsprozesse eine gewichtige Rolle, weil sich durch diese die lokalen Spezifika einer Planungskultur herausbilden. So gelten (kollektive) Lernprozesse als Bedingung für Innovationen und Veränderungen in den Arten und Weisen des Planens (vgl. Reimer 2013: 4655 f.). Annäherungsprozessen kommt dabei insbesondere in den kommunikativ-kooperativen Handlungsbereichen der Planung eine große Bedeutung $\mathrm{zu}$, in denen unterschiedliche Akteursgruppen mit ihren jeweiligen Haltungen und Orientierungen, Organisationsformen, Rollen- und Aufgabenverständnissen sowie Traditionen aufeinandertreffen (vgl. auch Selle 1994: 63 f.; Othengrafen/Sondermann 2015: 21). In diesem Sinne sind auch die (lokalen) gesellschaftlichen Kontexte und Dynamiken (z. B. gesellschaftlicher Werte- 
wandel, soziale Bewegungen, Initiativen und Proteste) von Bedeutung, da diese Lern- und Anpassungsprozesse auslösen und befördern können (vgl. auch Haumann 2015; Hölzl/ Nuissl 2015).

\subsection{Fazit}

Durch die Untersuchung einer empirisch beobachtbaren Planungskultur konnte in diesem Beitrag ein eigenes Planungskulturmodell entwickelt und zugleich gezeigt werden, welchen Mehrwert kulturelle Ansätze in der Planungswissenschaft haben: Der Fokus wurde auf spezifische Handlungsweisen der Akteure und die zugrunde liegenden Sinnsysteme gelenkt. Am Fallbeispiel Düsseldorf verdeutlicht heißt dies beispielsweise, dass sich die aktuelle Form kooperativer Stadtgrünentwicklung unter anderem aus der Gartenstadt-Tradition, langjährigen Erfahrungen in der Zusammenarbeit von Verwaltung und Zivilgesellschaft sowie gemeinsamen Haltungen und Orientierungen heraus erklären lässt. Dabei zeigt diese Arbeit im Ergebnis, dass nicht einzelne Elemente (wie handlungsleitende Denkweisen oder sichtbare Artefakte) entscheidend sind, sondern deren Zusammenspiel: Lokale Planungskulturen institutionalisieren sich in den jeweiligen kontextspezifischen Sinnsystemen, die sich durch Kommunikations-, Lern- und Annäherungsprozesse zwischen den beteiligten Akteursgruppen über die Zeit herausgebildet haben, deren Handeln leiten und sich in den spezifischen Arten und Weisen des Planens zeigen.

\section{Referenzen}

\section{Dokumente}

LHD 70/6/2010: Niederschrift über die Sitzung des Ausschusses für öffentliche Einrichtungen am 30.08.2010; 3.d - Antworten auf die Anfrage der Ratsfraktion Bündnis 90/Die Grünen zum Thema „Baumpatenschaften“; Landeshauptstadt Düsseldorf, 9-10.

LHD 70/39/2012: Vorlage zur Anfrage an den Vorsitzenden des Ausschusses für öffentliche Einrichtungen der Ratsfraktion Bündnis 90/Die Grünen zu „Urbane Gärten in Düsseldorf“; Landeshauptstadt Düsseldorf.

LHD 19/57/2012: Vorlage zur Anfrage an die Vorsitzende des Ausschusses für Umweltschutz der SPD-Ratsfraktion zum Thema „Positiveffekte der urbanen Agrikultur“; Landeshauptstadt Düsseldorf.

LHD 19/8/2012: Niederschrift über die Sitzung des Ausschusses für Umweltschutz am 15.11.2012; 3.a - Antworten auf die Anfrage der SPD-Ratsfraktion zum Thema „Positiveffekte der urbanen Agrikultur"; Landeshauptstadt Düsseldorf, 4-5.

LHD 70/8/2012: Niederschrift über die Sitzung des Ausschusses für öffentliche Einrichtungen am 12.11.2012; 3.a - Antworten auf die Anfrage der Ratsfraktion Bündnis 90/Die Grünen zum Thema „Urban Gardening“; Landeshauptstadt Düsseldorf, 4-5.

LHD 70/1/2015: Informationsvorlage. Politisches Controlling: Beschlüsse des Ausschusses für öffentliche Einrichtungen in der Zeit vom 01.07.2014 - 31.12.2014; Landeshauptstadt Düsseldorf.
LHD 70/5/2015: Information zu „Urban Gardening“ - Informationsvorlage für den Ausschuss für öffentliche Einrichtungen am 09.02.2015; Landeshauptstadt Düsseldorf.

LHD 176/221/2015: Antrag an den Bezirksbürgermeister des Stadtbezirks 6 der CDU-Fraktion, der FDP-Fraktion und der Fraktion BÜNDNIS 90/DIE GRÜNEN: Grundstück zur Nutzung für Urban Gardening (Gemeinschaftsgärten) vom 14.10.2015; Landeshauptstadt Düsseldorf.

\section{Literatur}

Anna, S.; Erben, R.; Görtz, W. (2010): Ökologische Stadt. In: Anna, S.; Erben, R.; Görtz, W. (Hrsg.): Ökologische Stadt. Düsseldorf, 6-13.

BBSR - Bundesinstitut für Bau-, Stadt- und Raumforschung (2015): Gemeinschaftsgärten im Quartier. Bonn. = BBSR-Online-Publikationen 12/2015.

Bläser, K.; Fox-Kämper, R.; Rawak, M.; Danielzyk, R.; Funke, L.; Sondermann, M. (2012): Urbanes Grün in der integrierten Stadtentwicklung. Strategien, Projekte, Instrumente. Düsseldorf.

BMVBS - Bundesministerium für Verkehr, Bau und Stadtentwicklung; BBR - Bundesamt für Bauwesen und Raumordnung (Hrsg.) (2007): Integrierte Stadtentwicklung als Erfolgsbedingung einer nachhaltigen Stadt. Hintergrundstudie zur „Leipzig Charta zur nachhaltigen europäischen Stadt" der deutschen Ratspräsidentschaft. Berlin, Bonn. = BBR-Online-Publikation 08/07.

Cultplan (2007): Cultural Differences in European Cooperation. Learning from INTERREG Practice. Wageningen.

DST - Deutscher Städtetag (2013): Beteiligungskultur in der integrierten Stadtentwicklung. Arbeitspapier der Arbeitsgruppe Bürgerbeteiligung des Deutschen Städtetages. Berlin, Köln.

Friedmann, J. (2005): Planning Cultures in Transition. In: Sanyal, B. (Hrsg.): Comparative Planning Cultures. New York, 29-44.

Fürst, D. (2007): Planungskultur. Auf dem Weg zu einem besseren Verständnis von Planungsprozessen? In: pnd-online 3, 1-15.

Glaser, B.; Strauss, A. (1967): The discovery of grounded theory: strategies for qualitative research. Chicago.

Haumann, S. (2015): Protest und Wertewandel: Zur Dynamik von Planungskulturen in den 1970er-Jahren. In: Othengrafen, F.; Sondermann, M. (Hrsg.): Städtische Planungskulturen im Spiegel von Konflikten, Protesten und Initiativen. Berlin, 87-110. = Planungsrundschau 23.

Healey, P. (2012): Performing Place Governance Collaboratively. Planning as a Communicative Process. In: Fischer, F.; Gottweis, H. (Hrsg.): The Argumentative Turn Revisited: Public Policy as Communicative Practice. Durham/London, 58-82.

Hetzel, A. (2001): Zwischen Poiesis und Praxis: Elemente einer kritischen Theorie der Kultur. Würzburg.

Hölzl, C.; Nuissl, H. (2015): Tragen Planungskonflikte zum Wandel der Planungskultur bei? Beobachtungen aus Santiago de Chile. In: Othengrafen, F.; Sondermann, M. (Hrsg.): Städtische Planungskulturen im Spiegel von Konflikten, Protesten und Initiativen. Berlin, 171-200. = Planungsrundschau 23.

Hopf, C. (2004): Qualitative Interviews - ein Überblick. In: Flick, U.; von Kardorff, E.; Steinke, I. (Hrsg.): Qualitative Forschung. Ein Handbuch. Reinbek, 349-360.

Ioannou, B.; Moran, N.; Sondermann, M.; Certoma, C.; Hardman, M. (2016): Grassroots Gardening Movements: Towards Cooperative Forms of Green Urban Development? In: Bell, S.; Fox-Kämper, R.; Keshavarz, N.; Benson, M.; Caputo, S.; Noori, S.; Voigt, A. (Hrsg.): Urban Allotment Gardens in Europe. Oxford, 62-89.

Keller, D. A.; Koch, M.; Selle, K. (1993a): „Entweder-Oder“ und „Und“. Erste Impressionen der Erkundungen zum Thema Planungskultur in vier Ländern. In: disP - The Planning Review 29, $115,74-84$. 
Keller, D. A.; Koch, M.; Selle, K. (Hrsg.) (1993b): Planungskulturen in Europa. Erkundungen in Deutschland, Frankreich, Italien und in der Schweiz. Darmstadt.

Knieling, J.; Othengrafen, F. (2009): En route to a theoretical model for comparative research on planning cultures. In: Knieling, J.; Othengrafen, F. (Hrsg.): Planning Cultures in Europe. Decoding Cultural Phenomena in Urban and Regional Planning. London, 39-62.

Knieling, J.; Othengrafen, F. (2015): Planning Culture - A Concept to Explain the Evolution of Planning Policies and Processes in Europe? In: European Planning Studies 23, 11, 2133-2147.

Läpple, D. (1992): Essay über den Raum. In: Häußermann, H.; Ibsen, D.; Krämer-Badoni, T.; Läpple, D.; Rodenstein, M.; Siebel, W. (Hrsg.): Stadt und Raum: soziologische Analysen. Pfaffenweiler, 157-207. = Stadt, Raum und Gesellschaft 1.

Landeshauptstadt Düsseldorf (Hrsg.) (2006): Gartenstadt Düsseldorf. Entwicklungen und Perspektiven innerstädtischer Grünflächen. Düsseldorf.

Landeshauptstadt Düsseldorf (2009): Soziale Stadt NRW, Düsseldorf Flingern/Oberbilk. Abschlussbericht. Düsseldorf. = Beiträge zur Stadtplanung und Stadtentwicklung in Düsseldorf 19.

Lange, C. (1987): Wohnungsnahes Grün - Düsseldorfer werden selbst aktiv. Erfahrungen mit Bürgerberatung. In: Das Gartenamt 36, 4, 240-243.

Levin-Keitel, M. (2015): Flusslandschaften in der Stadt. Einblicke in die empirische Erforschung von lokalen Planungskulturen. In: pnd-online 2, 1-13.

Levin-Keitel, M.; Sondermann, M. (2015): Räumliches Planen als kulturelles Handeln: Planungskultur als analytischer Ansatz. In: Othengrafen, F.; Sondermann, M. (Hrsg.): Städtische Planungskulturen im Spiegel von Konflikten, Protesten und Initiativen. Berlin, 31-58. = Planungsrundschau 23.

Löw, M. (2001): Raumsoziologie. Frankfurt am Main.

Lossau, J. (2007): Räume von Bedeutung. Spatial turn, cultural turn und Kulturgeographie. In: Csáky, M.; Leitgeb, C. (Hrsg.): Kommunikation - Gedächtnis - Raum. Kulturwissenschaften nach dem ,Spatial Turn“. Bielefeld, 29-43.

Mackrodt, U. (2015): Cui bono? Die Pioniernutzungen auf dem Tempelhofer Feld in Berlin zwischen Partizipation, Stadtmarketing und Protest. In: Othengrafen, F.; Sondermann, M. (Hrsg.): Städtische Planungskulturen im Spiegel von Konflikten, Protesten und Initiativen. Berlin, 277-302. = Planungsrundschau 23.

Markowitz, I. (1987): Die Gartenstadt Düsseldorf. In: Das Gartenamt $36,3,206-216$.

Marx, S.-P. (2007): Stadtplanung zwischen Umbruch und Kontinuität. In: Hamedinger, A.; Breitfuss, A.; Dangschat, J. S.; Frey, O. (Hrsg.): Strategieorientierte Planung im kooperativen Staat. Wiesbaden, 87-101.

Nikolaïdou, S.; Klöti, T.; Tappert, S.; Drilling, M. (2016): Urban Gardening and Green Space Governance: Towards New Collaborative Planning Practices. In: Urban Planning 1, 1, 5-19.

Nuissl, H.; Heinrichs, D. (2011): Fresh Wind or Hot Air - Does the Governance Discourse Have Something to Offer to Spatial Planning? In: Journal of Planning Education and Research 31, 1, 4759.

Othengrafen, F. (2010): Spatial Planning as Expression of Culturised Planning Practices. The Examples of Helsinki, Finland and Athens, Greece. In: Town Planning Review 81, 1, 83-110.

Othengrafen, F. (2012): Uncovering the Unconscious Dimensions of Planning. Using Culture as a Tool to Analyse Spatial Planning Practices. Farnham.

Othengrafen, F.; Reimer, M. (2013): The embeddedness of planning in cultural contexts: theoretical foundations for the analysis of dy- namic planning cultures. In: Environment and Planning A 45, 6 , 1269-1284.

Othengrafen, F.; Sondermann, M. (2015): Konflikte, Proteste, Initiativen und die Kultur der Planung - Stadtentwicklung unter demokratischen Vorzeichen? In: Othengrafen, F.; Sondermann, M. (Hrsg.): Städtische Planungskulturen im Spiegel von Konflikten, Protesten und Initiativen. Berlin, 7-30. = Planungsrundschau 23.

Peer, C.; Sondermann, M. (2016): Planungskultur als neues Paradigma in der Planungswissenschaft. In: disP - The Planning Review (im Erscheinen)

Reckwitz, A. (2000): Die Transformation der Kulturtheorien. Zur Entwicklung eines Theorieprogramms. Weilerswist.

Reckwitz, A. (2005): Kulturelle Differenzen aus praxeologischer Perspektive: Kulturelle Globalisierung jenseits von Modernisierungstheorie und Kulturessentialismus. In: Srubar, I.; Renn, J.; Wenzel, U. (Hrsg.): Kulturen Vergleichen. Sozial- und kulturwissenschaftliche Grundlagen und Kontroversen. Wiesbaden, 92-111.

Reimer, M. (2013): Planning Cultures in Transition: Sustainability Management and Institutional Change in Spatial Planning. In: Sustainability $5,11,4653-4673$.

Reimer, M.; Blotevogel, H. H. (2012): Comparing Spatial Planning Practice in Europe: A Plea for Cultural Sensitization. In: Planning Practice \& Research 27, 1, 7-24.

Reuter, W. (2000): Zur Komplementarität von Diskurs und Macht in der Planung. In: disP - The Planning Review 36, 141, 4-16.

Riegler, J. (2003): Aktuelle Debatten zum Kulturbegriff. Wien. = Working Papers der Kommission für Sozialanthropologie, Österreichische Akademie der Wissenschaften, Reihe A: Lokale Identitäten und überlokale Einflüsse 2.

Rosol, M. (2010): Public participation in post-Fordist urban green space governance: The case of community gardens in Berlin. In: International Journal of Urban and Regional Research 34, 3, 548563.

Sanyal, B. (Hrsg.) (2005): Comparative planning cultures. New York.

Selle, K. (1994): Was ist bloß mit der Planung los? Erkundungen auf dem Weg zum kooperativen Handeln. Ein Werkbuch. Dortmund. $=$ Dortmunder Beiträge zur Raumplanung 69 .

Selle, K. (2007): Stadtentwicklung und Bürgerbeteiligung. Auf dem Weg zu einer kommunikativen Planungskultur? Alltägliche Probleme, neue Herausforderungen. In: Informationen zur Raumentwicklung 1, 63-71.

Siebel, W. (2009): Die Welt lebenswerter machen. Stadtplanung als Gesellschaftspolitik. In: Mittelweg 36 - Zeitschrift des Hamburger Instituts für Sozialforschung 18, 6, 26-48.

Sondermann, M. (2014): Local Cultures of Urban Gardening and Planning in Germany. In: Keshavarz, N.; Fox-Kämper, R. (Hrsg.): Urban Allotment Gardens in European Cities - Future, Challenges and Lessons Learned (COST Action TU1201). Aachen, 15-32.

Sondermann, M. (2015): Zivilgesellschaftliches Engagement und die kulturelle Dimension kooperativer Stadtgrünentwicklung am Beispiel Hannovers. In: Neues Archiv für Niedersachsen 1, 98-111.

Steffler, H.-H.; Funke, K.-H.; Potratz, C.; Heckhausen, E.; Stoesling, C. (2006): 20 Jahre Ökotop Heerdt e.V. - Die Chronologie des Ökotops von 1986 bis 2006. Düsseldorf.

Stiftung Schloss und Park Benrath (2014): Elisabeths Garten - Gemüseanbau in der Stadt. http://www.elisabethsgarten.de (21.09.2016).

Strauss, A.; Corbin, J. (2010): Grounded theory: Grundlagen qualitativer Sozialforschung. Weinheim.

Suitner, J. (2014): Cultures of Image Construction. Approaching Planning Cultures as a Factor in Urban Image Production. In: European Spatial Research and Policy 21, 1, 39-52.

Wessel, K. (1996): Empirisches Arbeiten in der Wirtschafts- und Sozialgeographie: Eine Einführung. Paderborn. 